\title{
Treatment Profile of Pediatric Inflammatory Bowel Disease in Saudi Arabia: Issues in Treatment Adherence
}

\author{
Mohammed Hasosah ${ }^{1, *}$, Mohammed El Mouzan ${ }^{2}$, Omar Saadah $^{3}$, Khalid Al-Saleem $^{4}$, \\ Abdulrahman Al-Hussaini ${ }^{5}$, Ali Al Mehaidib ${ }^{4}$, Badr Al Saleem ${ }^{5}$, Khalid Alquair ${ }^{6}$, Kevan Jacobson ${ }^{7}$ \\ ${ }^{1}$ Department of Pediatrics, Division of Gastroenterology, King Saud Bin Abdulaziz University for Health Sciences, National Guard \\ Hospital, Saudi Arabia \\ ${ }^{2}$ Department of Pediatrics, Division of Gastroenterology, King Khalid University Hospital, King Saud University, Saudi Arabia \\ ${ }^{3}$ Department of Pediatrics, Division of Gastroenterology, King Abdulaziz University Hospital, King Abdulaziz University, Saudi Arabia \\ ${ }^{4}$ Department of Pediatrics, Division of Gastroenterology, King Faisal Specialist Hospital and Research Center, Saudi Arabia \\ ${ }^{5}$ Division of Pediatric Gastroenterology, Specialized Children's Hospital, King Fahad Medical City, King Saud bin Abdulaziz University \\ for Health Sciences, Saudi Arabia \\ ${ }^{6}$ Division of Pediatric Gastroenterology, King salman military hospital Tabuk, Saudi Arabia \\ ${ }^{7}$ Department of Pediatric Gastroenterology, British Colombia Children's Hospital, University of British Columbia, Canada
}

Copyright (C) 2015 by authors, all rights reserved. Authors agree that this article remains permanently open access under the terms of the Creative Commons Attribution License 4.0 International License

\begin{abstract}
Background: Inflammatory bowel disease (IBD), with its 3 subgroups: Crohn's disease (CD), ulcerative colitis (UC), and indeterminate colitis (IC), is a chronic relapsing inflammatory disorder of the gastrointestinal (GI) tract. Medication adherence is particularly problematic in pediatric IBD. To date this has not been studied in the Middle East. Objectives: The aim of this study was to describe the treatment modalities of IBD and to evaluate adherence to treatment in a population-based cohort of Saudi children. Methods: A cross-sectional study was conducted in all regions of Saudi Arabia. All

Saudi Arabia. Further research in IBD is required to determine the reasons for nonadherence/ poor adherence in the pediatric IBD population so that strategies can be devised to reduce it. Key Points: Medication adherence is particularly problematic in pediatric inflammatory bowel disease (IBD). We found that steroids and 5- Amino salicylic acids are the most widely used medications in pediatric IBD patients. Nonadherence occurs most frequently with 5-ASA, steroids and thiopurines in Crohns. These data should help health care providers quantify the burden of IBD among children.
\end{abstract} participating centers were asked to provide information on IBD medications and adherence to prescribed therapies. Results: 354 children with IBD were identified from our database. The age at diagnosis ranged from 1 to 14 years, with 145 (41\%) diagnosed with UC, 195 (55\%) with CD and $14(4 \%)$ with IC. The most common drugs therapy used in IBD was corticosteroids in CD and 5-ASA in UC. Patients with UC were treated with 5 -ASA significantly more than patients with $\mathrm{CD}(76 \%$ vs. $62 \%$; $\mathrm{P}=0.004)$. In contrast, patients with $\mathrm{CD}$ were treated with infliximab significantly more than patients with $\mathrm{UC}(22 \%$ vs. $6 \% ; \mathrm{P}=0.000)$. Overall, $15.8 \%$ of patients were non-adherent with patients with $\mathrm{CD}$ demonstrating a higher level of nonadherence, although this was not significantly different from UC patients $(11 \%$ vs. $3.8 \%$; $\mathrm{P}>0.05$ ). Poor adherence to specific drugs was reported significantly more in $\mathrm{CD}$ than $\mathrm{UC}$ patients; mesalamine $(p=0.022)$, steroids $(p=0.021)$ and thiopurines $(p=0.006)$. Conclusion: Poor adherence occurs most frequently in $\mathrm{CD}$ patients and with 5-ASA, corticosteroids and thiopurines. These data can be used to help care providers and policy makers such as the Ministry Of Health define and quantify the burden of IBD among children in
Keywords Inflammatory Bowel Disease, Treatment Adherence

\section{Introduction}

Inflammatory bowel disease (IBD), with its 3 subgroups: Crohn's disease (CD), ulcerative colitis (UC), and indeterminate colitis (IC), is a chronic relapsing inflammatory disorder of the gastrointestinal (GI) tract. IBD can affect children and adolescents of all ages, and as many as $25 \%$ of all IBD cases manifest before the age of 20 years. [1]

IBD management often requires long-term treatment based on the following; a combination of drugs to control the disease, disease location and phenotype, complications, individual symptomatic response and tolerance to medical intervention.

$\mathrm{CD}$ is the most prevalent form of IBD in children in the 
western region of Saudi Arabia and is comparable with that reported in other Saudi regions [2, 3]. El Mouzan et al, showed that the incidence of pediatric IBD in all regions of Saudi Arabia has increased significantly over time [4].

Medication adherence is particularly problematic in pediatric IBD because onset often occurs in adolescence [5]. In clinical practice, medication non-adherence occurs in $30 \%$ to $45 \%$ of patients with IBD [6], significantly increases the risk of experiencing a flare and is associated with higher overall health care costs $[7,8]$. The causes of medication nonadherence in IBD are multi-factorial. Evidence suggests that being young males, taking multiple medications , altering the dosing regimen and poor communication between the physician and the patient are contribution factors to medication nonadherence in IBD $[6,8]$.

To date, medication adherence in pediatric IBD has not been studied in Saudi Arabia and the Middle East. The aim of this study was to describe the treatment modalities of IBD and evaluate the adherence to treatments in a population-based cohort of Saudi children.

\section{Methods}

\subsection{Data Source}

This multicenter retrospective cohort study evaluated the initial treatment of children with IBD followed by pediatric gastroenterologists at seven tertiary centers in all regions of Saudi Arabia. Since 2003, the Pediatric IBD Registry has enrolled and followed newly diagnosed children $(<16$ years of age) with IBD, recording incidence, demographic information, treatments received and disease complications [4].

\subsection{Patient Selection}

All patients enrolled in the registry between January 2003 and December 2012 in all regions of Saudi Arabia were evaluated. The patients were identified either by computerized hospital medical or personal records. The diagnosis of IBD was based on clinical grounds, endoscopic evaluation, histological assessment of mucosal biopsy specimens and radiological findings (small bowel barium follow-through) in accordance with the Porto criteria. For each patient, the following data was recorded: diagnosis; year of diagnosis; age at diagnosis; gender; family history; symptoms at presentation; duration of symptoms before diagnosis; localization and extent of disease, severity, and clinical behavior; laboratory results; imaging findings; endoscopy findings; histopathology findings and extraintestinal manifestations. All participating centers were asked to review all IBD medications. Medications were divided into 5 classes: calcineurin inhibitors (tacrolimus or cyclosporine), biologics (infliximab and adalimumab), immunomodulators (methotrexate, azothioprine, 6-mercaptopurine), 5- Aminosalicylic acid (5-ASA) (mesalamine, balsalazide, sulfasalazine) and corticosteroids (budesonide, prednisolone). All participating centers were asked to provide information on drug adherence (very good, good and poor). A database form, completed by all participating centers, was designed specifically for this study and reviewed by two gastroenterologists (MH and AS).

\subsection{Ethical Considerations}

This report is part of the research project entitled, "Characteristics of inflammatory bowel disease in Saudi children" that was approved by the Institutional Review Board of the College of Medicine (No. 10/2647/IRB), King Saud University, Riyadh, KSA.

\subsection{Statistical Methods}

Statistical analyses were performed using Stata/IC 12.12011 software (Stata Corp LP, College Station, TX). The percentage of each drug therapy in IBD participants was determined. X2 and Fisher exact tests were used to compare categorical variables among groups. Student $t$ tests were used to compare continuous variables among groups. The results were considered significant if $\mathrm{P}<0.05$.

\section{Results}

We identified 354 children with IBD from our database. The age range of the population was 1014 years with 145 (41\%) diagnosed with UC, 195 (55\%) with CD and 14 (4\%) with IC.

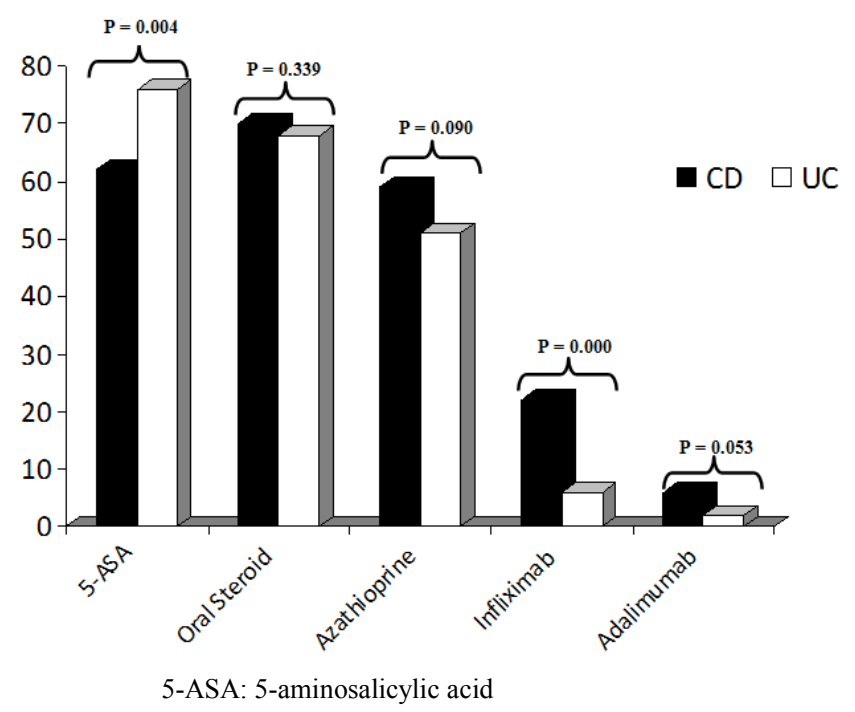

Figure 1. Type of IBD Medications

Table 1. Immunosuppressive and Biological therapies in IBD

\begin{tabular}{|c|c|c|c|}
\hline Disease & Thiopurines & Biological & P value \\
\hline UC (188) & $95(51 \%)$ & $14(8 \%)$ & 0.000 \\
\hline CD (373) & $219(59 \%)$ & $105(28 \%)$ & 0.000 \\
\hline
\end{tabular}




\subsection{Treatment Regimen}

The most common drugs therapy used in this IBD population was steroids (Prednisolone) in CD and 5-ASA (mesalamine; pentasa and asacol) in UC. $70 \%$ of CD patients were treated with oral steroids, $62 \%$ with 5 -ASA, $59 \%$ with thiopurines, $22 \%$ with infliximab and $6 \%$ with adalimumab. In UC patients, $76 \%$ received 5-ASA; other medications are shown in figure 1 . Patients with UC were significantly more likely to be treated with 5-ASA than patients with $\mathrm{CD}(76 \%$ vs. $62 \% ; \mathrm{P}=0.004)$. In contrast, patients with $\mathrm{CD}$ were significantly more likely to be treated with infliximab than patients with UC $(22 \%$ vs. $6 \% ; \mathrm{P}=0.000)$. No significant differences were observed between the UC and CD groups with respect to prescription of other IBD therapies including steroids, thiopurines and adalimumab. Drugs therapies including methotrexate, cyclosporine, tacrolimus and antibiotics and enteral nutrition were not reported in our study. Immunosuppressive therapies (thiopurines), were prescribed in $51 \%$ of patients with UC and $59 \%$ of patients with $\mathrm{CD}$, and $8 \%$ of $\mathrm{UC}$ and $28 \%$ of $\mathrm{CD}$ patients were exposed to biological therapies; $\mathrm{p}=0.000$ (Table 1).

\subsection{Non-adherence}

Overall, $15.8 \%(53 / 336)$ of patients were nonadherent to IBD therapies. Poor adherence according to the IBD type was seen more frequently in patients with $\mathrm{CD}$, although there was no significant difference in medication nonadherence between CD and UC (11\% vs. 3.8\%; P>0.05). Table 2 .

Poor adherence according to type of drug was reported significantly more in the CD than UC group. This included the following: mesalamine $(p=0.022)$, steroids $(p=0.021)$ and thiopurines $(\mathrm{p}=0.006)$. Drug compliance according to medication type is shown in table 3. Our data showed that $38.7 \%$ of patients were good adherent and $45.5 \%$ were very good adherent regardless of IBD type $(\mathrm{P}<0.05)$.

No data was available regarding the mean time between IBD diagnosis and start of immunosuppressive and biological therapies. No data was available regarding the duration of taking medicines. We could not link the type of drug therapy to disease status (mild, moderate, severe and refractory). No data was available regarding the patterns of IBD medication use, variation by patient age, gender, and geographic residence.

Table 2. Drug Compliance According to Disease Type

\begin{tabular}{|c|c|c|c|c|c|}
\hline \multirow{2}{*}{ Drug Compliance } & \multicolumn{3}{|c|}{ Diagnosis } & \multirow{2}{*}{ Total } & \multirow{2}{*}{ P value } \\
\cline { 2 - 5 } & UC & CD & IC & & 0.010 \\
\hline Very Good & $63(18.8 \%)$ & $83(24.7 \%)$ & $7(2.1 \%)$ & $153(45.5)$ & 0.000 \\
\hline Good & $66(19.6 \%)$ & $58(17.3 \%)$ & $6(1.8 \%)$ & $130(38.7 \%)$ & 0.300 \\
\hline Poor & $13(3.8 \%)$ & $37(11 \%)$ & $3(1 \%)$ & $53(15.8 \%)$ & 0 \\
\hline
\end{tabular}

Table 3. Drug Compliance According to Medication Type

\begin{tabular}{|c|c|c|c|c|}
\hline \multirow{2}{*}{ Drugs } & \multicolumn{3}{|c|}{ Diagnoses } & \multirow[b]{2}{*}{$P$ value } \\
\hline & UC & CD & IC & \\
\hline Mesalamine: N (\%) & $130(100)$ & $148(100)$ & $13(100)$ & \multirow{4}{*}{0.022} \\
\hline Very good & $53(41)$ & $66(44.6)$ & $6(46)$ & \\
\hline Good & $65(50)$ & $50(33.8)$ & $5(39)$ & \\
\hline Poor & $12(9)$ & $32(21.6)$ & $2(15)$ & \\
\hline Steroids: N (\%) & $132(100)$ & $141(100)$ & $14(100)$ & \multirow{4}{*}{0.021} \\
\hline Very good & $56(42)$ & $64(45)$ & $5(36)$ & \\
\hline Good & $63(48)$ & $45(32)$ & $6(43)$ & \\
\hline Poor & $13(10)$ & $32(23)$ & $3(21)$ & \\
\hline Thiopurines: N (\%) & $108(100)$ & $131(100)$ & $7(100)$ & \multirow{4}{*}{0.006} \\
\hline Very good & $38(35)$ & $64(49)$ & $4(57)$ & \\
\hline Good & $59(55)$ & $41(31)$ & $2(29)$ & \\
\hline Poor & $11(10)$ & $26(20)$ & $1(14)$ & \\
\hline Infliximab: N (\%) & $72(100)$ & $59(100)$ & $4(100)$ & \multirow{4}{*}{0.268} \\
\hline Very good & $24(33)$ & $30(51)$ & $1(25)$ & \\
\hline Good & $39(54)$ & $22(37)$ & $2(50)$ & \\
\hline Poor & $9(13)$ & $7(12)$ & $1(25)$ & \\
\hline Adalimumab: N (\%) & $4(100)$ & $19(100)$ & $2(100)$ & \multirow{4}{*}{0.514} \\
\hline Very good & $0(0)$ & $6(31.5)$ & $0(0)$ & \\
\hline Good & $3(75)$ & $7(37)$ & $1(50)$ & \\
\hline Poor & $1(25)$ & $6(31.5$ & $1(50)$ & \\
\hline
\end{tabular}




\section{Discussion}

In this multicenter retrospective study covering seven regions of Saudi Arabia, we determined treatment modalities of childhood IBD and medication nonadherenace.

We report here that patients with IBD were commonly prescribed steroids and 5-ASA for induction and maintenance treatment. This is similar to the findings in a study examining intercenter variation in initial management of children with Crohn's disease [9]. Rosen et al reported that the most common medications used in IBD population were immunomodulators for CD and 5-ASA for UC [10]. With regard to immunosuppressive and biological therapies use, our study showed a high rate of immunosuppressive exposure in children with IBD. More than half of patients used thiopurines. Although these treatments generally lead to better disease control and mucosal healing, they also increase the risk of hepatosplenic Tcell lymphoma and other unintended effects $[11,12]$. Careful population-based longitudinal cohort studies are needed to fully evaluate the risks and benefits of this evolving medical paradigm in the pediatric IBD population. We also found that thiopurines were commonly used in CD (59\%) and UC (51\%), although the use of biologic agents has increased $(28 \%)$ in this cohort. The majority of our IBD subjects on biologics were on these agents as monotherapy. However, since this was not a longitudinal study, we cannot comment on whether these agents were used as first-line treatment or because these patients had been refractory to other agents.

Measuring adherence is challenging. There is no error-free way to assess adherence and currently no gold standard exists [13].We chose clinician estimate of adherence rather than a self-reporting method such as direct interview or focused questionnaire because the overall accuracy of clinician prediction is estimated at $65 \%$ [14]. A number of studies have documented rates of medication nonadherence in pediatric IBD ranging from $38 \%-66 \%$, depending on the reporter, medication type, and method of assessment (e.g., objective methods versus subjective self-reporting methods) $[15,16,17]$. In our study, $15.8 \%$ of patients were nonadherent to medication. This low percentage could be explained by not documenting and measuring adherence by the medication adherence reporting scale (MARS) and Morisky scale which have been proposed for use in measuring adherence in IBD [18, 19, 20].

Understanding the causes of nonadherence is important and is reported in the IBD literature [19]. In our pediatric IBD population the reasons for medication nonadherence were not reported, but possible explanations of nonadherenace include the following; medication side effects, child feeling well, belief medication was ineffective, longer disease duration or medication duration and the local pharmacy did not stock the medication.

Most studies, regardless of the definition of nonadherence used, have reported that there is no difference in adherence according to disease type [6]. We report here that patients with $\mathrm{CD}$ were more likely to be nonadherent to medication than patients with UC. This is contrary to the findings of a study using self-reported adherence to all of the IBD medications, which suggested nonadherence was more common in UC [19]. Conversely, our data showed that $38 \%-45 \%$ of patients were fully adherent to their medications. The lack of objective measure of adherence such as counting pills or checking prescription refills in our study might explain the high adherence. Medication adherence is extremely important as optimizing compliance rates across treatments may help improve patient outcomes [21].

There are some strategies for optimizing patient adherence especially pediatric IBD. Communication is a key component in improving patient adherence and a good physician-patient relationship has been shown to be associated with higher medication-adherence rates [20]. We can suggest to implement an education program for pediatric IBD in Saudi Arabia. This program includes the following; 1) patient information and support. Patients and their families can log on to their websites to learn more about the disease. eg, Saudi Arabia Society of Pediatric Gastroenterology, Hepatology and Nutrition (SASPGHAN). 2) Practical aide memoires. Patients could also be prompted to take their medications via simple pill-taking cues, such as placing pills close to something they use daily, e.g. the toothpaste, breakfast table, toys, etc. In addition, telephone support, postal reminders, setting alarms on watches/mobile phones, have been suggested.

The strengths of this study include the population-based design to ensure near complete capture of all IBD cases diagnosed and treated in all parts of Saudi Arabia; complete participation of the most pediatric gastroenterologists in Saudi Arabia; the geographic situation of Saudi Arabia and availability of pediatric gastroenterologists making it unlikely that patients with IBD would be seek treatment outside of our catchment area; and the relative abundance and access to pediatric gastroenterologists in Saudi Arabia making it unlikely for children to be diagnosed and followed by adult gastroenterologists.

This study had several limitations. Firstly, the uses of a clinician estimate of adherence as a sole measure to assess adherence. Secondly, our population comprised primarily of children and adolescents followed at IBD referral centers throughout Saudi Arabia and may not be representative of the general pediatric IBD population [4].

\section{Conclusions}

We implemented a population-based pediatric IBD case identification system covering the entire regions of Saudi Arabia, and characterized treatment modalities of IBD. This study demonstrated that steroids and 5-ASA are the most widely used medications in pediatric IBD patients. Nonadherence occurs most frequently with 5-ASA, steroids and thiopurines in CD. These data should help health care providers and policy makers such as the Ministry Of Health 
quantify the burden of IBD among children in Saudi Arabia. Further research in the IBD is required to determine the reasons for nonadherence in pediatrics so that strategies can be developed and implemented to reduce it.

\section{Acknowledgements}

The authors extend their appreciations to the Deanship of Scientific Research at King Saud University for funding this work through Research Group No. RG-1436-007.

\section{Author Contributions}

All authors contributed equally to the conception and design of the study, data collection, and critical revision of the manuscript, and agree to be accountable for all aspects of the work.

\section{Conflict of Interests}

The authors declare that there are no conflict of interests regarding the publication of this paper.

\section{REFERENCES}

[1] IBD Working Group of the European Society for Paediatric Gastroenterology, Hepatology and Nutrition (ESPGHAN). Inflammatory bowel disease in children and adolescents: recommendations for diagnosis-the Porto Criteria. J Pediatr Gastroenterol Nutr 2005; 41: 1-7.

[2] El Mouzan MI, Al Mofarreh MA, Assiri AM, Hamid YH, Al Jebreen AM, Azzam NA. Presenting features of childhoodonset inflammatory bowel disease in the central region of Saudi Arabia. Saudi Med J 2012; 33: 423-428.

[3] Hasosah MY, Sukkar GA, Alsahafi AF, Kutbi SY, Alzabn AA, Jacobson K. Pediatric inflammatory bowel disease in the western region of Saudi Arabia. A retrospective analysis. Saudi Med J 2013 ;34(6):651-3.

[4] El Mouzan MI, Saadah O, Al-Saleem K, Al Edreesi M, Hasosah $\mathrm{M}$ et al. Incidence of pediatric inflammatory bowel disease in saudi arabia: a multicenter national study. Inflamm Bowel Dis. 2014;20 (6):1085-90.

[5] Sandler RS, Eisen GM. Epidemiology of inflammatory bowel disease. In: Kirsner JB, ed. Inflammatory Bowel Disease. London, UK: W. B. Saunders; 2000:89-112.

[6] Jackson CA, Clatworthy J, Robinson A, et al. Factors associated with nonadherence to oral medication for inflammatory bowel disease: a systematic review. Am J Gastroenterol. 2010;105:525-539.

[7] Kane S, Huo D, Aikens J, et al. Medication nonadherence and the outcomes of patients with quiescent ulcerative colitis. $\mathrm{Am}$ J Med. 2003;114: 39-43.

[8] Kane S, Shaya F. Medication non-adherence is associated with increased medical health care costs. Dig Dis Sci. 2008;53:1020-1024.

[9] Kappelman MD, Bousvaros A, Hyams J et al. Intercenter variation in initial management of children with Crohn's disease. Inflamm Bowel Dis. 2007;13(7):890-5.

[10] Rosen D, Kathy-Hoffstadter-Thal, Bao R, et al. Analysis of current treatments used in clinical practice in a pediatric summer camp population for children with inflammatory bowel disease. Inflamm Bowel Dis. 2012 ;18(10):1818-24.

[11] Rosh JR, Gross T, Mamula P, et al. Hepatosplenic T-cell lymphoma in adolescents and young adults with Crohn's disease: a cautionary tale? Inflamm Bowel Dis. 2007;13:1024-1030.

[12] Mackey AC, Green L, Liang LC, et al. Hepatosplenic T cell lymphoma associated with infliximab use in young patients treated for inflammatory bowel disease. $J$ Pediatr Gastroenterol Nutr. 2007;44:265-267.

[13] Rapoff MA. Adherence to Pediatric Medical Regimens. New York: Kluwer Academic; 1999.

[14] Finney JW, Hook RJ, Friman PC, et al. The overestimation of adherence to pediatric medical regimens. Child Health Care. 1993;22:297-304.

[15] Hommel KA, Davis CM, Baldassano RN. Objective versus subjective assessment of oral medication adherence in pediatric inflammatory bowel disease. Inflamm Bowel Dis. 2009; 15:589-593.

[16] Mackner LM, Crandall,WV. Oral medication adherence in pediatric inflammatory bowel disease. Inflamm Bowel Dis. 2005;11:1006-1012.

[17] Oliva-Hemker MM, Abadom V, Cuffari C, et al. Nonadherence with thiopurine immunomodulator and mesalamine medications in children with Crohn disease. $J$ Pediatr Gastroenterol Nut. 2007;44:180-184.

[18] Rigby D. Adherence assessment tools: drugs don't work when they are not taken. Australian $J$ Pharmacy. 2007;88:32-37.

[19] Ediger JP, Walker JR, Graff L, et al. Predictors of medication adherence in inflammatory bowel disease. $\mathrm{Am} \mathrm{J}$ Gastroenterol. 2007;102:1417-1426.

[20] Sewitch MJ, Abrahamowicz M, Barkun A, et al. Patient nonadherence to medication in inflammatory bowel disease. Am J Gastroenterol. 2003;98:1535-1544.

[21] Hawthorne AB, Rubin G, Ghosh S. Review article: medication nonadherence in ulcerative colitis - strategies to improve adherence with mesalazine and other maintenance therapies. Aliment Pharmacol Ther. 2008;27:1157-1166. 